\title{
MOVING INTERFACIAL GRIFFITH CRACK BETWEEN BONDED DISSIMILAR MEDIA
}

\author{
S. MUKHERJEE AND S. DAS
}

Received 27 October 2003 and in revised form 29 October 2004

The plane strain problem of determining strain energy release rate, crack energy, and crack-opening displacement (COD) for a moving Griffith crack at the interface of two dissimilar orthotropic half-planes is considered. The problem is reduced to a pair of singular integral equations of second kind which have finally been solved by using Jacobi polynomials. Graphical plots of the strain energy release rate, crack energy, and crackopening displacement for the problem in different particular cases are presented.

\section{Introduction}

Structures in real life not only experience tensions but encounter forces like shear and torsional loading. Cracks, therefore, are likely to be subjected to both tension and shear resulting in mixed mode cracking. The combined effect of tension and shear gives rise to mixture of modes I and II. Several investigators like Nuismer [15], Erdogan and Sih [7], Sih [16], and so forth have considered mixed mode fracture problems.

Problems of interfacial Griffith cracks in orthotropic media had been studied by Das and Patra [2], Das et al. [3], Dhaliwal et al. [4], He et al. [9], Erdogan and Wu [8], Nisitani et al. [14], Liou and Sung [13], Lee [12], and so forth. In this paper, the determination of strain energy release rate, and crack energy, crack-opening displacement around a moving Griffith crack at the interface of two dissimilar orthotropic half-planes under mixed-mode loading is considered. The problem is reduced to solving a pair of singular integral equations of second kind which are ultimately solved by the technique described by Erdogan [6] and by Erdogan and Wu [8]. Finally, numerical results for normalized stain energy release rate, crack energy, and crack-opening displacement have been calculated for some particular cases. Obtained results are also presented graphically. It has also been observed that the locus of the fracture is an elliptic contour corresponding to the critical crack velocity.

\section{Formulation of the problem}

We consider the plane elastodynamic problem in orthotropic half plane $1(-\infty<X<\infty$, $0 \leq Y<\infty)$ bonded to a dissimilar orthotropic half-plane $2(-\infty<X<\infty,-\infty<Y \leq 0)$ 
with a moving Griffith crack of finite length situated at the interface of the two materials. The principal axes of the materials coincide with the Cartesian coordinate axes. As in Yoffe model [19], it is assumed that the cracks are propagating with constant velocity " $c$ " and without change in length along the positive $X$ axis. This assumption is justified by the fact that the stress distribution close to one end of a crack is not influenced by its distance from the other. In what follows and in the sequel, the quantities with subscripts $i=1,2$ refer to those for the half-planes (1) and (2), respectively.

Under the assumption of plane strain in an orthotropic medium, equations of motion for the displacement fields are

$$
\begin{aligned}
& C_{11}^{(i)} \frac{\partial^{2} U^{(i)}}{\partial X^{2}}+C_{66}^{(i)} \frac{\partial^{2} U^{(i)}}{\partial Y^{2}}+\left(C_{12}^{(i)}+C_{66}^{(i)}\right) \frac{\partial^{2} V^{(i)}}{\partial X \partial Y}=\rho^{(i)} \frac{\partial^{2} U^{(i)}}{\partial t^{2}}, \\
& C_{22}^{(i)} \frac{\partial^{2} V^{(i)}}{\partial Y^{2}}+C_{66}^{(i)} \frac{\partial^{2} V^{(i)}}{\partial X^{2}}+\left(C_{12}^{(i)}+C_{66}^{(i)}\right) \frac{\partial^{2} U^{(i)}}{\partial X \partial Y}=\rho^{(i)} \frac{\partial^{2} V^{(i)}}{\partial t^{2}},
\end{aligned}
$$

where $t$ is the time, $\rho^{(i)}$ and $C_{j k}^{(i)}, i=1,2$, are respective densities and elastic constants of the half-planes (1) and (2). Applying the Gallilean transformation $x=X-c t, y=Y$, $t=t$, equations (2.1) become independent of $t$ and reduce to

$$
\begin{aligned}
& \left(C_{11}^{(i)}-c^{2} \rho^{(i)}\right) \frac{\partial^{2} u^{(i)}}{\partial x^{2}}+C_{66}^{(i)} \frac{\partial^{2} u^{(i)}}{\partial y^{2}}+\left(C_{12}^{(i)}+C_{66}^{(i)}\right) \frac{\partial^{2} v^{(i)}}{\partial x \partial y}=0 \\
& \left(C_{66}^{(i)}-c^{2} \rho^{(i)}\right) \frac{\partial^{2} v^{(i)}}{\partial x^{2}}+C_{22}^{(i)} \frac{\partial^{2} v^{(i)}}{\partial y^{2}}+\left(C_{12}^{(i)}+C_{66}^{(i)}\right) \frac{\partial^{2} u^{(i)}}{\partial x \partial y}=0
\end{aligned}
$$

where $u^{(i)}(x, y)=U^{(i)}(X, Y, t), v^{(i)}(x, y)=V^{(i)}(X, Y, t)$, and $i=1,2$.

The stress displacement relations are

$$
\begin{aligned}
& \sigma_{x x}^{(i)}=C_{11}^{(i)} \frac{\partial u^{(i)}}{\partial x}+C_{12}^{(i)} \frac{\partial v^{(i)}}{\partial y}, \\
& \sigma_{y y}^{(i)}=C_{22}^{(i)} \frac{\partial v^{(i)}}{\partial y}+C_{12}^{(i)} \frac{\partial u^{(i)}}{\partial x}, \\
& \sigma_{x y}^{(i)}=C_{66}^{(i)}\left(\frac{\partial u^{(i)}}{\partial y}+\frac{\partial v^{(i)}}{\partial x}\right), \quad i=1,2 .
\end{aligned}
$$

It is assumed that the crack, now defined by $|x| \leq a, y= \pm 0$, is opened by internal tractions $-p_{1}(x)$ and $-p_{2}(x)$ in such a way as to guarantee the symmetry requirements. Then 
the boundary conditions at the interface $y=0$ are

$$
\begin{gathered}
\sigma_{y y}^{(1)}(x, 0)=\sigma_{y y}^{(2)}(x, 0)=-p_{1}(x), \quad|x| \leq a, \\
\sigma_{x y}^{(1)}(x, 0)=\sigma_{x y}^{(2)}(x, 0)=-p_{2}(x), \quad|x| \leq a, \\
\sigma_{y y}^{(1)}(x, 0)=\sigma_{y y}^{(2)}(x, 0), \quad|x|>a, \\
\sigma_{x y}^{(1)}(x, 0)=\sigma_{x y}^{(2)}(x, 0), \quad|x|>a, \\
u^{(1)}(x, 0)=u^{(2)}(x, 0), \quad|x|>a, \\
v^{(1)}(x, 0)=v^{(2)}(x, 0), \quad|x|>a .
\end{gathered}
$$

In addition, all components of stress and displacement vanish at remote distance from the crack. For a subsonic propagation, it is assumed that the Mach numbers $M_{j}^{(i)}=c / v_{j}^{(i)}$ $(i, j=1,2)$ are less than one. $v_{1}^{(i)}=\sqrt{C_{11}^{(i)} / \rho^{(i)}}$ and $v_{2}^{(i)}=\sqrt{C_{66}^{(i)} / \rho^{(i)}}(i=1,2)$ represent velocities of elastic waves. The former is called the velocity of dilatation waves and the latter is known as velocity of shear waves of the mediums 1 and 2 .

\section{Solution of the problem}

An appropriate integral solution of (2.2) is taken as

$$
\begin{aligned}
& u^{(i)}(x, y)=\int_{0}^{\infty} A^{(i)}(s, y) \sin s x d s, \\
& v^{(i)}(x, y)=\int_{0}^{\infty} B^{(i)}(s, y) \cos s x d s,
\end{aligned}
$$

where $A^{(i)}$ and $B^{(i)}$ for the half-planes (1) and (2) are given by

$$
\begin{aligned}
& A^{(i)}(s, y)=A_{1}^{(i)}(s) e^{(-1)^{i} \gamma_{1}^{(i)} s y}+A_{2}^{(i)}(s) e^{(-1)^{i} \gamma_{2}^{(i)} s y}, \\
& B^{(i)}(s, y)=(-1)^{i}\left[B_{1}^{(i)}(s) e^{(-1)^{i} \gamma_{1}^{(i)} s y}+B_{2}^{(i)}(s) e^{(-1)^{i} \gamma_{2}^{(i)} s y}\right]
\end{aligned}
$$

in which $\gamma_{1}^{(i)}, \gamma_{2}^{(i)}\left(<\gamma_{1}^{(i)}\right)$ are the positive roots of the equation

$$
\begin{aligned}
C_{66}^{(i)} C_{22}^{(i)} \gamma^{4}+ & {\left[\left(C_{12}^{(i)}+C_{66}^{(i)}\right)^{2}+C_{22}^{(i)}\left(C_{11}^{(i)}-c^{2} \rho^{(i)}\right)-C_{66}^{(i)}\left(C_{66}^{(i)}-c^{2} \rho^{(i)}\right)\right] \gamma^{2} } \\
+ & \left(C_{66}^{(i)}-c^{2} \rho^{(i)}\right)\left(C_{11}^{(i)}-c^{2} \rho^{(i)}\right)=0
\end{aligned}
$$

and $A_{j}^{(i)}(s)(i=1,2$ and $j=1,2)$ are arbitrary functions and $B_{j}^{(i)}(s)$ are related to $A_{j}^{(i)}(s)$ by

$$
B_{j}^{(i)}=-\frac{\alpha_{j}^{(i)}}{\gamma_{j}^{(i)}} A_{j}^{(i)},
$$


where

$$
\alpha_{j}^{(i)}=\frac{C_{11}^{(i)}-c^{2} \rho^{(i)}-C_{66}^{(i)}\left(\gamma_{j}^{(i)}\right)^{2}}{\left(C_{12^{(i)}}+C_{66}^{(i)}\right)} .
$$

Now,

$$
\begin{aligned}
& \sigma_{y y}^{(i)}(x, y)=\int_{0}^{\infty}\left[\left(C_{12}^{(i)}-\alpha_{1}^{(i)} C_{22}^{(i)}\right) A_{1}^{(i)} e^{(-1)^{i} \gamma_{1}^{i} s y}+\left(C_{12}^{(i)}-\alpha_{2}^{(i)} C_{22}^{(i)}\right) A_{2}^{(i)} e^{(-1)^{i} y_{2}^{(i)} s y}\right] s \cos s x d s, \\
& \sigma_{x y}^{(i)}(x, y)=(-1)^{i} C_{66}^{(i)} \int_{0}^{\infty}\left[\frac{\beta_{1}^{(i)}}{\gamma_{1}^{(i)}} A_{1}^{(i)} e^{(-1)^{i} \gamma_{1}^{(i)} s y}+\frac{\beta_{2}^{(i)}}{\gamma_{2}^{(i)}} A_{2}^{(i)} e^{(-1)^{i} \gamma_{2}^{(i)} s y}\right] s \sin s x d s,
\end{aligned}
$$

where

$$
\beta_{j}^{(i)}=\alpha_{j}^{(i)}+\left[\gamma_{j}^{(i)}\right]^{2}, \quad i=1,2 ; j=1,2 .
$$

Boundary conditions (2.4) and (2.6) give rise to

$$
\eta_{1}^{(1)} A_{1}^{(1)}+\eta_{2}^{(1)} A_{2}^{(1)}=\eta_{1}^{(2)} A_{1}^{(2)}+\eta_{2}^{(2)} A_{2}^{(2)},
$$

where

$$
\eta_{j}^{(i)}=C_{12}^{(i)}-\alpha_{j}^{(i)} C_{22}^{(i)}, \quad i=1,2 ; j=1,2 .
$$

Boundary conditions (2.5) and (2.7) give rise to

$$
-\mu_{1}^{(1)} A_{1}^{(1)}-\mu_{2}^{(1)} A_{2}^{(1)}=\mu_{1}^{(2)} A_{1}^{(2)}+\mu_{2}^{(2)} A_{2}^{(2)},
$$

where

$$
\mu_{j}^{(i)}=C_{66}^{(i)} \frac{\beta_{j}^{(i)}}{\gamma_{j}^{(i)}}, \quad i=1,2 ; j=1,2 .
$$

Equations (3.8) and (3.10) lead to

$$
\begin{aligned}
& A_{1}^{(2)}=\frac{\mu_{1}^{(1)} \eta_{2}^{(2)}+\mu_{2}^{(2)} \eta_{1}^{(1)}}{\eta_{1}^{(2)} \mu_{2}^{(2)}-\eta_{2}^{(2)} \mu_{1}^{(2)}} A_{1}^{(1)}+\frac{\mu_{2}^{(1)} \eta_{2}^{(2)}+\mu_{2}^{(2)} \eta_{2}^{(1)}}{\eta_{1}^{(2)} \mu_{2}^{(2)}-\eta_{2}^{(2)} \mu_{1}^{(2)}} A_{2}^{(1)}, \\
& A_{2}^{(2)}=\frac{\mu_{1}^{(2)} \eta_{1}^{(1)}+\eta_{1}^{(2)} \mu_{1}^{(1)}}{\mu_{1}^{(2)} \eta_{2}^{(2)}-\mu_{2}^{(2)} \eta_{1}^{(2)}} A_{1}^{(1)}+\frac{\mu_{1}^{(2)} \eta_{2}^{(1)}+\eta_{1}^{(2)} \mu_{2}^{(1)}}{\mu_{1}^{(2)} \eta_{2}^{(2)}-\mu_{2}^{(2)} \eta_{1}^{(2)}} A_{2}^{(1)} .
\end{aligned}
$$

Boundary conditions (2.8) and (2.9) together with (3.12) yield

$$
\begin{array}{ll}
\int_{0}^{\infty}\left[w_{11} A_{1}^{(1)}(s)+w_{12} A_{2}^{(1)}(s)\right] \sin s x d s=0, & |x|>a, \\
\int_{0}^{\infty}\left[w_{12} A_{1}^{(1)}(s)+w_{22} A_{2}^{(1)}(s)\right] \cos s x d s=0, & |x|>a,
\end{array}
$$


where

$$
\begin{aligned}
& w_{11}=1-\frac{\mu_{1}^{(1)} \eta_{2}^{(2)}+\mu_{2}^{(2)} \eta_{1}^{(1)}}{\eta_{1}^{(2)} \mu_{2}^{(2)}-\eta_{2}^{(2)} \mu_{1}^{(2)}}+\frac{\mu_{1}^{(2)} \eta_{1}^{(1)}+\mu_{1}^{(1)} \eta_{1}^{(2)}}{\eta_{1}^{(2)} \mu_{2}^{(2)}-\eta_{2}^{(2)} \mu_{1}^{(2)}}, \\
& w_{12}=1-\frac{\mu_{2}^{(1)} \eta_{2}^{(2)}+\mu_{2}^{(2)} \eta_{2}^{(1)}}{\eta_{1}^{(2)} \mu_{2}^{(2)}-\eta_{2}^{(2)} \mu_{1}^{(2)}}+\frac{\mu_{1}^{(2)} \eta_{1}^{(1)}+\mu_{2}^{(1)} \eta_{1}^{(2)}}{\eta_{1}^{(2)} \mu_{2}^{(2)}-\eta_{2}^{(2)} \mu_{1}^{(2)}}, \\
& w_{21}=\frac{\alpha_{1}^{(1)}}{\gamma_{1}^{(1)}}+\frac{\alpha_{1}^{(2)}}{\gamma_{1}^{(2)}}\left(\frac{\mu_{1}^{(1)} \eta_{2}^{(2)}+\mu_{2}^{(2)} \eta_{1}^{(1)}}{\eta_{1}^{(2)} \mu_{2}^{(2)}-\eta_{2}^{(2)} \mu_{1}^{(2)}}\right)-\frac{\alpha_{2}^{(2)}}{\gamma_{2}^{(2)}}\left(\frac{\mu_{1}^{(2)} \eta_{1}^{(1)}+\eta_{1}^{(2)} \mu_{1}^{(1)}}{\eta_{1}^{(2)} \mu_{2}^{(2)}-\eta_{2}^{(2)} \mu_{1}^{(2)}}\right), \\
& w_{22}=\frac{\alpha_{2}^{(1)}}{\gamma_{2}^{(1)}}+\frac{\alpha_{1}^{(2)}}{\gamma_{1}^{(2)}}\left(\frac{\mu_{2}^{(1)} \eta_{2}^{(2)}+\mu_{2}^{(2)} \eta_{2}^{(1)}}{\eta_{1}^{(2)} \mu_{2}^{(2)}-\eta_{2}^{(2)} \mu_{1}^{(2)}}\right)-\frac{\alpha_{2}^{(2)}}{\gamma_{2}^{(2)}}\left(\frac{\mu_{1}^{(2)} \eta_{2}^{(1)}+\eta_{1}^{(2)} \mu_{2}^{(1)}}{\eta_{1}^{(2)} \mu_{2}^{(2)}-\eta_{2}^{(2)} \mu_{1}^{(2)}}\right) .
\end{aligned}
$$

Now set

$$
\begin{aligned}
& w_{11} A_{1}^{(1)}(s)+w_{12} A_{2}^{(1)}(s)=\frac{1}{s} \int_{0}^{a} f_{1}(t) \cos s t d t, \\
& w_{21} A_{1}^{(1)}(s)+w_{22} A_{2}^{(1)}(s)=\frac{1}{s} \int_{0}^{a} f_{2}(t) \sin s t d t,
\end{aligned}
$$

where $f_{1}(t)$ is an even function of $t$ and $f_{2}(t)$ is an odd function of $t$. It is observed that (3.13) is indentically satisfied under the conditions

$$
\int_{-a}^{a} f_{i}(t) d t=0, \quad i=1,2
$$

Now form (3.15), $A_{j}^{(1)}(s), j=1,2$, are calculated as

$$
\begin{aligned}
& A_{1}^{(1)}(s)=\frac{W_{22}}{s} \int_{0}^{a} f_{1}(t) \cos s t d t-\frac{W_{12}}{s} \int_{0}^{a} f_{2}(t) \sin s t d t, \\
& A_{2}^{(1)}(s)=\frac{W_{11}}{s} \int_{0}^{a} f_{2}(t) \sin s t d t-\frac{W_{21}}{s} \int_{0}^{a} f_{1}(t) \cos s t d t,
\end{aligned}
$$

where

$$
\begin{array}{ll}
W_{11}=\frac{w_{11}}{w_{11} w_{22}-w_{12} w_{21}}, & W_{12}=\frac{w_{12}}{w_{11} w_{22}-w_{12} w_{21}}, \\
W_{21}=\frac{w_{21}}{w_{11} w_{22}-w_{12} w_{21}}, & W_{22}=\frac{w_{22}}{w_{11} w_{22}-w_{12} w_{21}} .
\end{array}
$$

Now substituting the values of $A_{k}^{(1)}(s)$ from (3.17) in the boundary condition (2.4) and (2.5), the following singular integral equations, for the determination of the unknown functions $f_{1}(t)$ and $f_{2}(t)$, we obtained

$$
\begin{array}{ll}
a_{1} f_{1}(x)-\frac{1}{\pi b_{1}} \int_{-a}^{a} \frac{f_{2}(t) d t}{t-x}=\frac{2}{\pi} p_{1}(x), & \text { for }|x| \leq a, \\
c_{1} f_{2}(x)-\frac{1}{\pi d_{1}} \int_{-a}^{a} \frac{f_{1}(t) d t}{t-x}=\frac{2}{\pi} p_{2}(x), & \text { for }|x| \leq a,
\end{array}
$$


where

$$
\begin{array}{ll}
a_{1}=-\left(\eta_{1}^{(1)} W_{22}-\eta_{2}^{(1)} W_{21}\right), & b_{1}=-\frac{1}{\left(\eta_{2}^{(1)} W_{11}-\eta_{1}^{(1)} W_{12}\right)}, \\
c_{1}=-\left(\mu_{1}^{(1)} W_{12}-\mu_{2}^{(1)} W_{11}\right), & d_{1}=\frac{1}{\left(\mu_{1}^{(1)} W_{22}-\mu_{2}^{(1)} W_{21}\right)} .
\end{array}
$$

Now, setting $x / a=Z$ and $t / a=T$, (3.20) becomes

$$
\begin{array}{ll}
a_{1} f_{1}(a Z)-\frac{1}{\pi b_{1}} \int_{-1}^{1} \frac{f_{2}(a T) d T}{T-Z}=\frac{2}{\pi} p_{1}(a Z), & |Z| \leq 1, \\
c_{1} f_{2}(a Z)-\frac{1}{\pi d_{1}} \int_{-1}^{1} \frac{f_{1}(a T) d T}{T-Z}=\frac{2}{\pi} p_{2}(a Z), & |Z| \leq 1 .
\end{array}
$$

As $a_{1}, b_{1}, c_{1}$, and $d_{1}$ depend on the material constants and the velocity of propagation $c$, the signs of the quantities may be any combination for different types of orthoropic materials. Varying " $c$ " such that the Mach numbers $M_{j}^{(i)}$ remain less than unity, if the signs of these quantities are all positive, then (3.20) can be expressed as

$$
\phi_{k}(Z)+\frac{1}{\pi i \varepsilon r_{k}} \int_{-1}^{1} \frac{\phi_{k}(T) d T}{T-Z}=-g_{k}(Z), \quad|Z|<1, k=1,2,
$$

where

$$
\begin{aligned}
\phi_{k}(Z) & =\sqrt{a_{1} b_{1}} f_{1}(a Z)+i r_{k} \sqrt{c_{1} d_{1}} f_{2}(a Z), \\
\varepsilon & =\sqrt{a_{1} b_{1} c_{1} d_{1}}, \quad r_{1}=1, \quad r_{2}=-1, \\
g_{k}(Z) & =\frac{2}{\pi}\left(\sqrt{\frac{b_{1}}{a_{1}}} p_{1}(a Z)+i r_{k} \sqrt{\frac{d_{1}}{c_{1}}} p_{2}(a Z)\right) .
\end{aligned}
$$

\section{Solution of the integral equations}

The solution of the integral equations in (3.23) may be assumed as (Erdogan [6])

$$
\phi_{k}(Z)=w_{k}(Z) \sum_{n=0}^{\infty} C_{k n} P_{n}^{\left(\alpha_{k}, \beta_{k}\right)}(Z)
$$

where $w_{k}(Z)=(1-Z)^{\alpha_{k}}(1+Z)^{\beta_{k}}, \alpha_{k}=-1 / 2+i w_{k}, \beta_{k}=-1 / 2-i w_{k}, w_{k}=w r_{k}, k=1,2$, $w=(1 / 2 \pi) \ln |(1+\varepsilon) /(1-\varepsilon)|$, and $C_{k n}$ are unknown constants. By virtue of (3.16), we have

$$
C_{k 0}=0, \quad k=1,2 .
$$


Using the result

$$
\begin{aligned}
& \frac{1}{\pi i} \int_{-1}^{1} w_{k}(T) P_{n}^{\left(\alpha_{k}, \beta_{k}\right)}(T) \frac{d T}{T-X} \\
& \quad=-\varepsilon r_{k} w_{k}(Z) P_{n}^{\left(\alpha_{k}, \beta_{k}\right)}(Z)+\frac{\sqrt{1-\varepsilon^{2}}}{2 i} P_{n-1}^{\left(-\alpha_{k},-\beta_{k}\right)}(Z), \quad-1<Z<1, \\
& \quad=\left(1-\varepsilon r_{k}\right)\left\lfloor w_{k}(Z) P_{n}^{\left(\alpha_{k}, \beta_{k}\right)}(Z)-G_{k n}^{\infty}(Z)\right\rfloor, \quad|z|>1,
\end{aligned}
$$

where $G_{k n}^{\infty}$ is the principal part of $w_{k}(Z) P_{n}^{\left(\alpha_{k}, \beta_{k}\right)}(Z)$ at infinity, the integral equations (3.23) with the aid of (4.1) give rise to

$$
\frac{\sqrt{1-\varepsilon^{2}}}{2 i \varepsilon r_{k}} \sum_{n=1}^{\infty} C_{k n} P_{n-1}^{\left(-\alpha_{k},-\beta_{k}\right)}(Z)=-g_{k}(Z), \quad k=1,2 .
$$

Multiplying both sides of $(4.4)$ by $w_{k}^{-1} \mathbf{P}_{n}^{\left(-\alpha_{k},-\beta_{k}\right)}(Z)$ and integrating with respect to $Z$ from -1 to 1 and using orthogonality relation, the values of unknowns $C_{k j}(k=1,2, j=$ $0,1,2, \ldots)$ are given by

$$
C_{k j+1}=\frac{-i \varepsilon r_{k} g_{k j}}{\sqrt{1-\varepsilon^{2}}} \frac{(j+1) ! \Gamma(j+2)}{\Gamma\left(j-a_{k}+1\right) \Gamma\left(j-\beta_{k}+1\right)},
$$

where $g_{k j}=\int_{-1}^{1} g_{k}(Z) w_{k}^{-1}(Z) P_{j}^{\left(-\alpha_{k},-\beta_{k}\right)}(Z) d Z$.

The stress intensity factors near the crack tip $x=a$ may be calculated as

$$
\begin{aligned}
\sqrt{\frac{b_{1}}{a_{1}}} K_{I}+i r_{k} \sqrt{\frac{d_{1}}{c_{1}}} K_{I I} & =L t_{x \rightarrow a+}(x-a)^{-\alpha_{k}}(x+a)^{-\beta_{k}}\left[\sqrt{\frac{b_{1}}{a_{1}}} \sigma_{y y}^{(1)}(x, 0)+i r_{k} \sqrt{\frac{d_{1}}{c_{1}}} \sigma_{x y}^{(1)}(x, 0)\right] \\
& =\frac{-a \pi i \sqrt{1-\varepsilon^{2}}}{2 \varepsilon r_{k}} \sum_{n=1}^{\infty} C_{k n} P_{n}^{\left(\alpha_{k}, \beta_{k}\right)}(1) .
\end{aligned}
$$

Considering $p_{1}(x)=p$ and $p_{2}(x)=0$, with $p$ being a constant, the stress intensity factors are calculated as

$$
K_{I}=-a p, \quad K_{I I}=-\frac{a p}{\pi} \sqrt{\frac{b_{1} c_{1}}{a_{1} d_{1}}} \ln \left|\frac{1+\sqrt{a_{1} b_{1} c_{1} d_{1}}}{1-\sqrt{a_{1} b_{1} c_{1} d_{1}}}\right| .
$$

The crack-opening displacement is given by the following equation:

$$
\Delta v(x, 0)=v^{(1)}(x, 0)-v^{(2)}(x, 0)=\frac{\pi a^{2} p b_{1}}{\sqrt{1-\varepsilon^{2}}} \frac{\left(1+\omega^{2}\right)}{\left(9+4 \omega^{2}\right)\left(1+4 \omega^{2}\right)}\left(1-\frac{x}{a}\right)^{2} .
$$


The strain energy release rate is calculated as (Erdogan and $\mathrm{Wu}[8]$ )

$$
\frac{G}{a p^{2}}=\frac{\sqrt{a_{1} b_{1} c_{1} d_{1}}}{2}\left[\frac{b_{1}}{a_{1}} K_{I}^{2}+\frac{d_{1}}{c_{1}} K_{I I}^{2}\right]
$$

The expression for crack energy is given by

$$
\begin{aligned}
W & =-\int_{-a}^{a} p_{1}(x)\left[v^{(1)}(x, 0)-v^{(2)}(x, 0)\right] d x \\
& =\frac{\pi}{2} \cdot \frac{b_{1}^{2} p^{2}}{\sqrt{1-\varepsilon^{2}}}\left(1+4 \omega^{2}\right) \operatorname{sech}(\pi w) .
\end{aligned}
$$

Over the crack, the distance apart of two surfaces is given by

$$
v^{(1)}(x, 0)-v^{(2)}(x, 0)=\operatorname{Im} \frac{\pi i \varepsilon a}{\sqrt{1-\varepsilon^{2}}}\left(\frac{a-x}{2 a}\right)^{1 / 2+i w}\left[1-\frac{3-2 i w}{9+4 w^{2}}\left(\frac{a-x}{2 a}\right)\right] .
$$

For this problem, the distances of two surfaces should be greater than or equal to zero, but near the ends of the crack the sign changes infinitely indicating that the upper and lower surfaces of the crack should wrinkle and overlap each other, which is physically impossible.

Since we are interested to evaluate the size of the region in which the overlapping occurs, we consider the crack surfaces first come to rest when

$$
\cos \left(w \ln \frac{a-x}{2 a}\right)=0
$$

Thus, contact first takes place at a distance $\delta$ from the ends of the crack, where

$$
w \ln \left(\frac{\delta}{2 a}\right)= \pm \frac{\pi}{2}
$$

and hence $\delta$ has a maximum value of

$$
\delta=2 a e^{-\pi / 2 w}
$$

\section{Numerical results and discussions}

As a particular case of the problem, the orthotropic materials are considered to be $\alpha$ uranium and beryllium. The elastic moduli $\left(10^{11}\right.$ dynes $\left./ \mathrm{cm}^{2}\right)$ and densities $\left(\mathrm{gm} / \mathrm{cm}^{3}\right)$ of the materials are taken as in Table 5.1 (Hearman [10]). 
Table 5.1

\begin{tabular}{cccrrrl}
\hline & $C_{11}$ & \multicolumn{1}{c}{$C_{22}$} & \multicolumn{1}{c}{$C_{66}$} & $C_{12}$ & \multicolumn{1}{c}{$\rho$} & \\
\hline$\alpha$-uranium & 21.47 & 19.36 & 7.43 & 4.65 & 19.07 & (Half plane (1)) \\
Beryllium & 31.48 & 36.49 & 11.24 & 8.88 & 1.80 & (Half plane (2)) \\
\hline
\end{tabular}

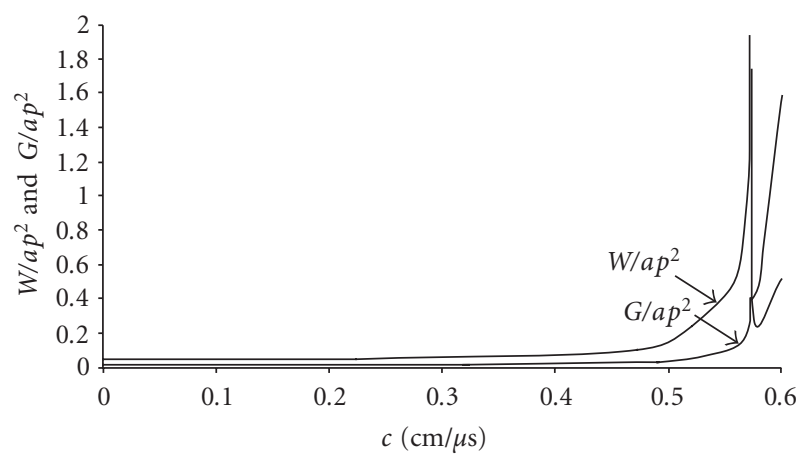

Figure 5.1. Plot of $W / a p^{2}$ and $G / a p^{2}$ versus $c$.

For a subsonic propagation considering the crack velocity $c=0(0.1) 0.6$, the strain energy release rate and crack energy are calculated at the crack tip $x=a$ (Figure 5.1). It is seen from Figure 5.1 that the strain energy release rate $\left(G / a p^{2}\right)$ increases up to $c=0.575$ and then this has oscillatory nature, which is expected as there is a change of propagation phase from subsonic to supersonic.

Similar oscillation phenomena have been observed by England [5], Williams [18], Sneddon and Lowengrub [17], and so forth. Since we have considered a subsonic propagation where $M_{j}^{(i)}<1$, that is, $c<\operatorname{Min}\left(v_{1}^{(1)}, v_{2}^{(1)}, v_{1}^{(2)}, v_{2}^{(2)}\right)$, we can conclude that, following Achenbach [1], Stoneley waves will be observed at the interface outside the crack surface and Rayleigh waves will be observed at the free surface of the crack. However, it may be noted that, at any point of the interface, Stonely waves will not propagate in all directions as noted by Johnson [11]. It is also seen from Figure 5.1 that initially the amplitude of crack energy $G / a p^{2}$ curve is less than that of $W / a p^{2}$ and it oscillates and intersects the second curve at the point $c=0.5736 \mathrm{~cm} / \mu \mathrm{s}$. (The Mach numbers at this crack velocity are $M_{1}^{(1)}=0.5406, M_{2}^{(1)}=0.9189, M_{1}^{(2)}=0.1372, M_{2}^{(2)}=0.2295$.) We know fracture occurs when $G \geq W$. Thus in this mixed mode II loading problem, the fracture condition is more likely to be

$$
\frac{K_{I}^{2}}{K_{I c}^{2}}+\frac{K_{I I}^{2}}{K_{I I c}^{2}}=1
$$

the locus of fracture being an ellipse (Figure 5.2), where $K_{I c}=\sqrt{\left(a_{1} / b_{1}\right) W_{c}}, K_{I I c}=$ $\sqrt{\left(c_{1} / d_{1}\right) W_{c}}$ are critical stress intensity factors, $W_{c}$ is the critical crack energy at $c=$ $0.5736 \mathrm{~cm} / \mu \mathrm{s}$. 


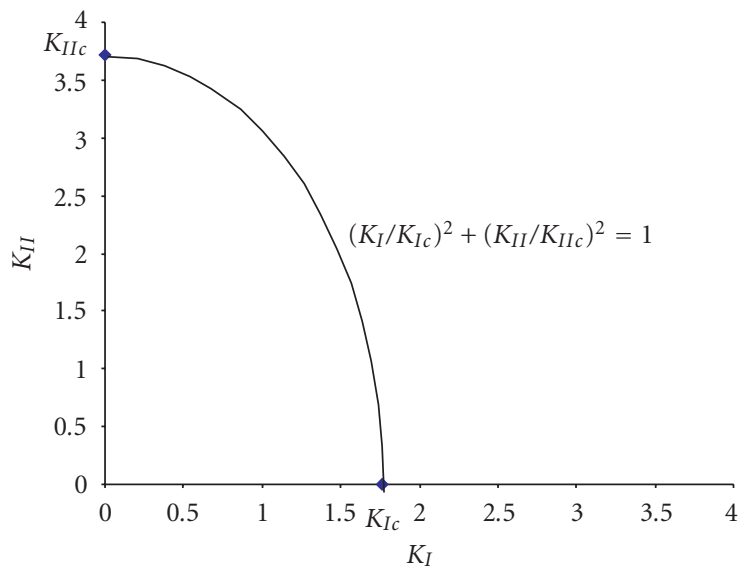

Figure 5.2. Combined mode fracture at $c=0.5736$.

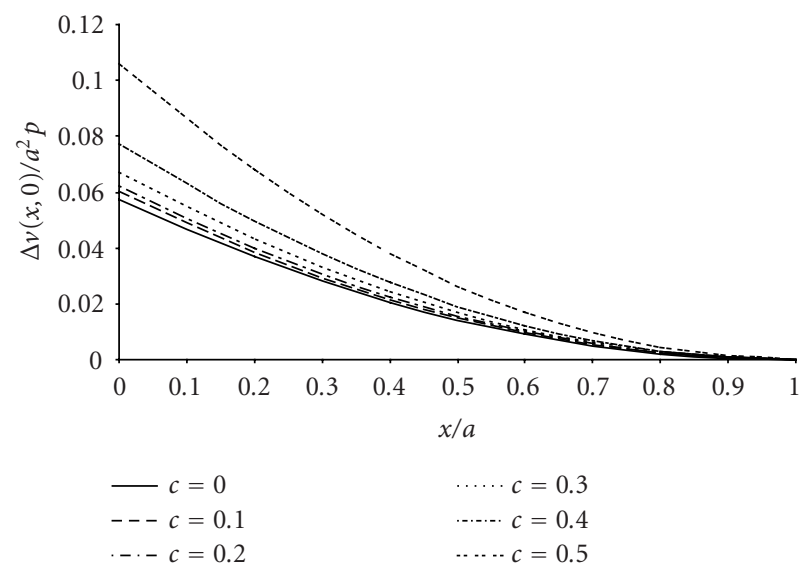

Figure 5.3. Plot of $\Delta v(x, 0) / a^{2} p$ versus $x / a$.

The crack-opening displacement (COD) $\Delta v(x, 0) / a^{2} p$ versus $x / a$ has been displayed graphically in Figure 5.3 for different $c$. It has been observed that the COD is maximum at the central position of the crack and decreases continuously to become zero at the tip of the crack.

\section{Acknowledgments}

The authors are grateful to the Department of Science and Technology, Government of West Bengal, India, for the financial support granted for the work. The authors express their sincere thanks to the referee for valuable suggestions in the improvement of the paper. 


\section{References}

[1] J. D. Achenbach, Wave Propagation in Elastic Solids, North-Holland Publishing, New York, 1973.

[2] S. Das and B. Patra, Stress intensity factors for moving interfacial crack between bonded dissimilar fixed orthotropic layers, Comput. \& Structures 69 (1998), no. 4, 459-472.

[3] S. Das, B. Patra, and L. Debnath, Stress intensity factors for a moving interfacial Griffith crack in composite media, Internat. J. Engrg. Sci. 37 (1999), no. 4, 453-475.

[4] R. S. Dhaliwal, H. S. Saxena, and J. G. Rokne, Elasticity: mathematical methods and applications, The Ian N. Sneddon 70th Birthday Volume, Ellis Horwood, Chester, 1990, pp. 59-86.

[5] A. H. England, A crack between dissimilar media, J. Appl. Mech. 28 (1965), 400-402.

[6] F. Erdogan, Approximate solutions of systems of singular integral equations, SIAM J. Appl. Math. 17 (1969), 1041-1059.

[7] F. Erdogan and G. C. Sih, On the crack extension in plates under plane loading and transverse shear, J. Basic Engng. 85 (1963), 519-527.

[8] F. Erdogan and Binghua Wu, Interface crack problems in layered orthotropic materials, J. Mech. Phys. Solids 41 (1993), no. 5, 889-917.

[9] W. He, R. S. Dhaliwal, and H. S. Saxena, Griffith crack at the interface of two orthotropic eleastic layers, Engrg. Fract. Mech. 41 (1992), 13-25.

[10] R. F. S. Hearman, An Introduction to Applied Anisotropic Elasticity, Oxford University Press, Oxford, 1961.

[11] W. W. Johnson, The propagation of Stoneley and Rayleigh waves in anisotropic elastic media, Bull. Seismol. Soc. Amer. 60 (1970), 1105-1122.

[12] K. H. Lee, Stress and displacement fields for propagating the crack along the interface of dissimilar orthotropic materials under dynamic Mode I and Mode II load, Trans. ASME J. Appl. Mech. 67 (2000), 223-228.

[13] J. Y. Liou and J. C. Sung, Singularities at the tip of a crack terminating normally at an interface between two orthotropic media, Trans. ASME J. Appl. Mech. 63 (1996), no. 2, 264-270.

[14] H. Nisitani, A. Simimoto, and H. Noguchi, Analysis of an interface crack based on the body method, Transactions of the Japan Society of Mechanical Engineers 59 (1993), 68-73.

[15] R. J. Nuismer, An energy release rate criterion for mixed mode fracture, Internat. J. Fracture 11 (1975), 245-250.

[16] G. C. Sih, Strain energy density factor applied to mixed mode crack problems, Internat. J. Fracture 10 (1974), 305-322.

[17] I. N. Sneddon and M. Lowengrub, Crack Problems in the Classical Theory of Elasticity, John Wiley \& Sons, New York, 1969.

[18] M. L. Williams, The stresses around a fault or crack in dissimilar media, Bull. Seismol. Soc. Amer. 49 (1959), 199-204.

[19] Elizabeth H. Yoffe, The moving Griffith crack, Philos. Mag. (7) 42 (1951), 739-750.

S. Mukherjee: Department of Mathematics, B. P. Poddar Institute of Management and Technology, Poddar Vihar, 137 V. I. P. Road, Calcutta 700052, West Bengal, India

S. Das: Department of Mathematics, B. P. Poddar Institute of Management and Technology, Poddar Vihar, 137 V. I. P. Road, Calcutta 700052, West Bengal, India

E-mail address: sananda_das@hotmail.com 


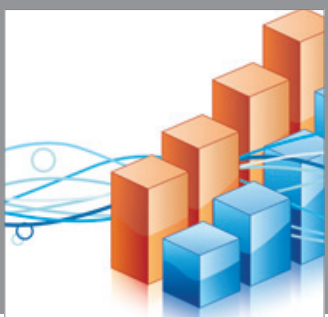

Advances in

Operations Research

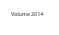

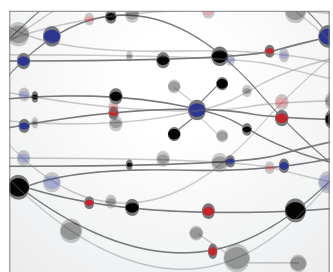

\section{The Scientific} World Journal
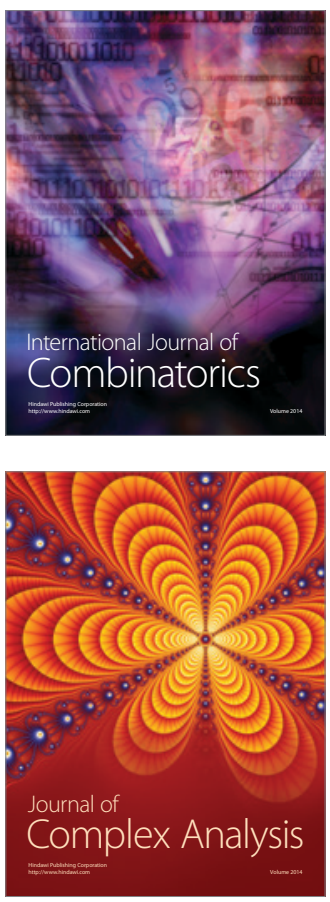

International Journal of

Mathematics and

Mathematical

Sciences
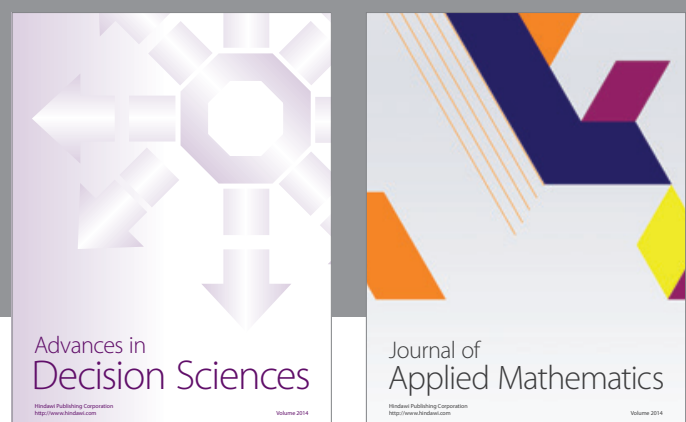

Journal of

Applied Mathematics
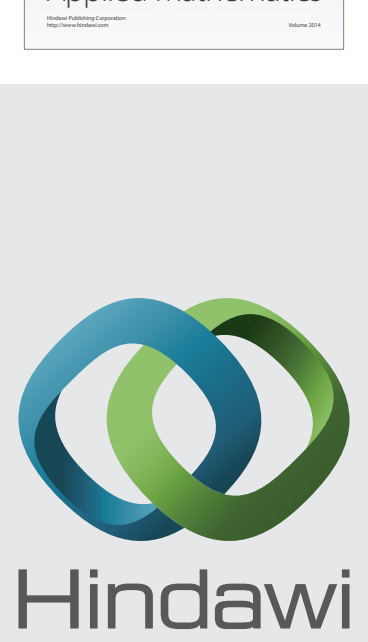

Submit your manuscripts at http://www.hindawi.com
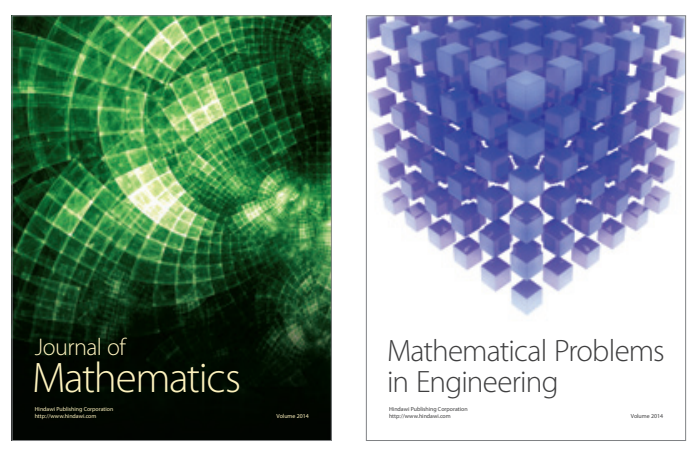

Mathematical Problems in Engineering
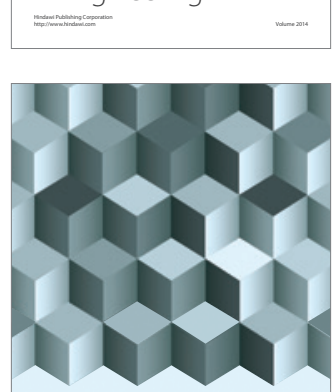

Journal of

Function Spaces
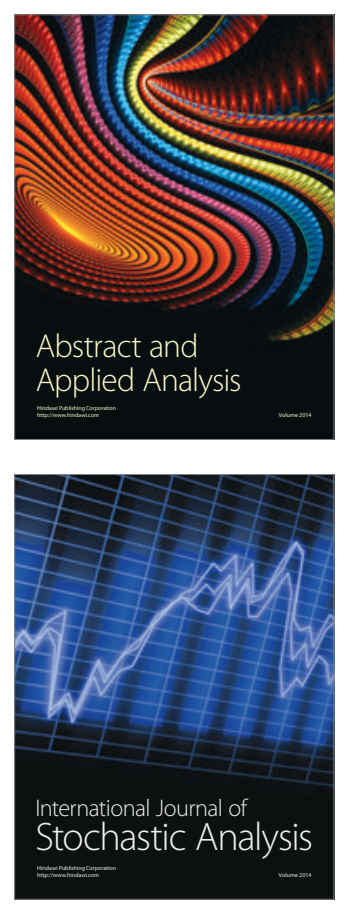

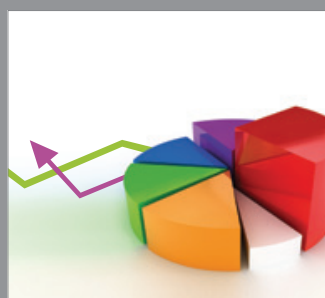

ournal of

Probability and Statistics

Promensencen
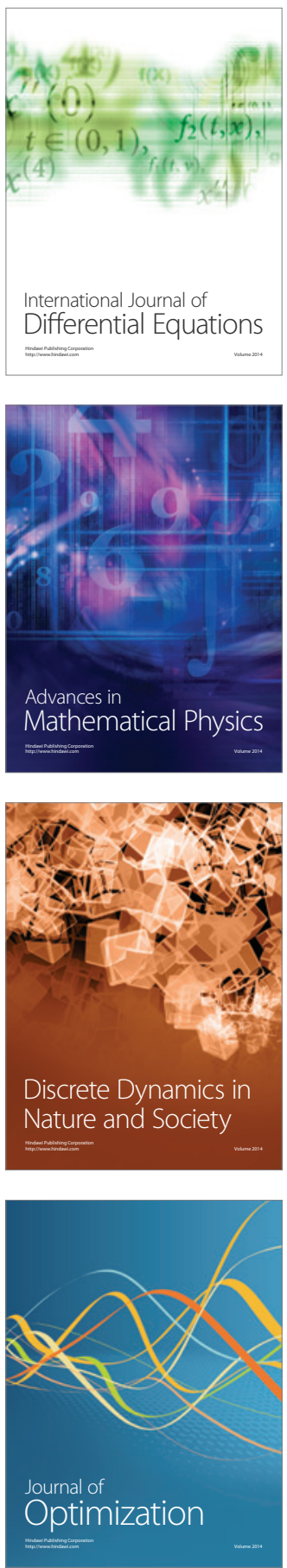